\title{
XLII. On quaternions; or on a new system of imaginaries in algebra
}

\section{Sir William Rowan Hamilton LL.D. M.R.I.A. F.R.A.S.}

To cite this article: Sir William Rowan Hamilton LL.D. M.R.I.A. F.R.A.S. (1849) XLII. On quaternions; or on a new system of imaginaries in algebra, Philosophical Magazine Series 3 , 34:229, 294-297, DOI: $10.1080 / 14786444908646233$

To link to this article: http://dx.doi.org/10.1080/14786444908646233

曲 Published online: 30 Apr 2009.

Submit your article to this journal $\sqsubset x$

Џ Article views: 2

Q View related articles $\square$

Citing articles: 1 View citing articles 5 
interest, and might lead to meteorological results of a remarkable nature.

I cannot conclude this abstract without drawing attention to the circumstance, that $\mathrm{M}$. Arago had already pointed out in 1820, shortly after CErsted's discovery, the possibility of acting upon the voltaic arc by this magnet, and the analogy which might result between this phænomenon and that of the aurora borealis.

XLII. On Quaternions; or on a Nerw System of Imaginaries in Algebra. By Sir William Rowan Hamilton, LL.D., M.R.I.A., F.R.A.S., Corresponding Member of the Institute of France, \&c., Andrewes' Professor of Astronomy in the University of Dublin, and Royal Astronomer of Ireland.

[Continued from vol. xxxiii. p. 60.]

65. IF we make

$$
\rho-\lambda=\lambda ; \rho \rho-\mu=\mu_{1} ; \rho-\lambda^{\prime}=\lambda_{1}^{\prime} ; \rho-\mu^{\prime}=\mu_{1}^{\prime} ; \quad .
$$

and in like manner, (see (106.), )

$$
\rho-\xi=-b^{2} v=\xi_{1} ; . . . . .
$$

and if we regard these five new vectors, $\lambda_{i}, \mu_{p}, \lambda_{l}^{\prime}, \mu_{i}^{\prime}$, and $\xi_{p}$, as lines which, being drawn from the centre $A$, terminate respectively in five new points, $\mathbf{L}_{l}, \mathbf{M}_{i}, \mathbf{L}_{j}^{\prime}, \mathbf{M}_{1}^{\prime}$, and $\mathrm{H}_{\mathrm{j}}$ while the vector $\rho$, drawn from the same centre $A$, still terminates in the point $E$, upon the surface of the ellipsoid; then the equations (113.), (114.), of art. 62, will give :

$$
\mathrm{T} \lambda_{1}=\mathrm{T} \mu_{1}=\mathrm{T} \lambda_{1}^{\prime}=\mathrm{T}_{\mu^{\prime}}=b ; . . .
$$

while the equations (101.) will enable us to write

$$
\frac{\lambda_{1}-\xi_{1}}{x}=\frac{\mu_{1}-\xi_{1}}{!}=\frac{\mu_{1}-\lambda_{1}}{1-x}=\mathrm{V}^{-1} 0 ; . .
$$

and in like manner, (see (112.),)

$$
\frac{\lambda_{1}^{\prime}-\xi_{1}}{x^{\prime}}=\frac{\mu_{1}^{\prime}-\xi_{1}}{!}=\frac{\mu_{1}^{\prime}-\lambda_{l}^{\prime}}{I^{\prime}-x^{\prime}}=\mathrm{V}^{-1} 0 ;
$$

this symbol $\mathrm{V}^{-1} 0$ denoting (as already explained) a scalar. We shall have also, by (84.), (89.),

$$
\frac{p-\lambda_{1}}{\imath-x}=\frac{\lambda}{\imath-x}=\mathrm{V}^{-1} 0 ; \frac{\rho-\mu_{1}}{x-\imath}=\frac{\mu}{x-\imath}=\mathrm{V}^{-1} 0 ;
$$

the scalars denoted by the symbol $\mathrm{V}^{-1} 0$ being not generally obliged to be equal to each other, and being, in these last 
equations (120.), respectively equal, by (86.), (91.), to those which have been denoted above by $h$ and $h^{\prime}$. In like manner, by (110.),

$$
\frac{\rho-\lambda_{l}^{\prime}}{\iota^{\prime}-x^{\prime}}=\frac{\lambda^{\prime}}{\iota^{\prime}-x^{\prime}}=\mathrm{V}^{-1} 0 ; \quad \frac{\rho-\mu_{l}^{\prime}}{x^{\prime}-\iota^{\prime}}=\frac{\mu^{\prime}}{x^{\prime}-\iota^{\prime}}=\mathrm{V}^{-1} 0 .
$$

And because, by (107.), $t^{\prime}$ has a scalar ratio to $x$, and $x^{\prime}$ has a scalar ratio to b, we may infer, from (118.), (119.), the existence of the two following other scalar ratios:

$$
\frac{\mu_{1}^{\prime}-\xi_{1}}{\lambda_{1}-\xi_{1}}=\mathrm{V}^{-1} 0 ; \frac{\lambda_{1}^{\prime}-\xi_{1}}{\mu_{1}-\xi_{1}}=\mathrm{V}^{-1} 0 . .
$$

Finally we may observe that, by (120.), (121.), there exist scalar ratios between certain others also of the foregoing vector-differences, and especially the following:

$$
\frac{\rho-\lambda_{1}}{\rho-\mu_{1}}=\mathrm{V}^{-1} 0 ; \frac{\rho-\lambda_{1}^{\prime}}{\rho-\mu_{1}^{\prime}}=\mathrm{V}^{-1} 0 . . .
$$

66. Proceeding now to consider the geometrical signification of the equations in the last article, we see first, from the equations (117.), that the four new points, $\mathrm{L}_{j}, \mathbf{M}_{l}, \mathrm{~L}_{l}^{\prime}, \mathbf{M}_{j}^{\prime}$, are all situated upon the surface of that mean sphere, which is described on the mean axis of the ellipsoid as a diameter; because the equation of that mean sphere has been already seen to be

$$
p^{2}+b^{2}=0 * \text { equation (i00.), article } 58 ;
$$

which may also be thus written, by the principles and notations of the calculus of quaternions:

$$
\mathrm{T}_{p}=b \text {. }
$$

From the relations (122.) it follows that the two chords $\mathbf{L}_{l} \mathbf{M}_{i}^{\prime}$ and $\mathbf{L}_{l}^{\prime} \mathrm{M}_{l}$, of this mean sphere, both pass through the point $\mathrm{H}$, of which the vector $\xi$ is assigned by the formula (116.); for

* This form of the equation of the sphere was published in the Philosophical Magazine for July 1846 ; and it is an immediate and a very easy consequence of that fundamental formula of the whole theory of Quaternions, namely

$$
i^{2}=j^{2}=l^{2}=i j k=-1,
$$

which was communicated under a slightly more developed form, to the Royal Irish Academy, on the 13th of November 1843. (See Phil. Mag. for July 1844.)

It may perhaps be thought not unworthy of curions notice hereafter, that after the publication of this form of the equation of the sphere, there should have been found in England, and in 1846, a person with any mathematical character to lose, who could profess publicly bis inability to distinguish the method of quaternions from that of couples; and who could thus confound the system of the present writer with those of Argand and of Français, of Mourey and of Warren. 
the first equation (122.) shows that the three vectors $\lambda_{1}, \mu_{1}^{\prime}, \xi_{p}$, which are all drawn from one common point, namely the centre $\Lambda$ of the ellipsoid, all terminate on one straight line; since otherwise the quotient of their differences, $\mu_{1}^{\prime}-\xi_{1}$ and $\lambda_{l}-\xi_{1}$, would be a quaternion*, of which the vector part would not be equal to zero: and in like manner, the second equation (122.) expresses that the three lines $\lambda_{l}^{\prime}, \mu_{i}, \xi_{j}$, all terminate on another straight line. 'The four-sided figure $\mathbf{L}_{i} M_{1} I_{l}^{\prime} M_{l}^{\prime}$ is therefore a plane quadrilateral, inscribed (generally) in a small circle of the mean sphere, and having the point $\mathrm{i}$ for the intersection of its second and fourth sides, $M_{l} L_{l}^{\prime}$ and $M_{l}^{\prime} L_{j}$, or of those two sides prolonged. And these two sides, having respectively the directions of $\mathrm{HM}_{i}$ and $\mathrm{HL}_{l}$, or of the vectordifferences $\mu_{1}-\xi_{1}$ and $\lambda_{1}-\xi_{1}$, are respectively parallel, by (118.), to the two fixed vectors, 1 and $x$; or (by what was shown in former articles), to the two cyclic normals, $\mathrm{AC}^{\prime}$ and Ac, of the original ellipsoid. 'The plane of the quadrilateral inscribed in the mean sphere is therefore constantly parallel to the principal plane $\mathbf{C A} \mathbf{c}^{\prime}$ of that ellipsoid, namely to the plane of the greatest and least axes, which contains those two cyclic normals. 'The first and third sides, $L_{t} M_{1}$ and $L_{l}^{\prime} M_{j}^{\prime}$, of the same inscribed quadrilateral, being in the directions of $\mu_{1}-\lambda_{1}$ and $\mu_{1}^{\prime}-\lambda_{1}^{\prime}$, are parallel, by (118.), (119.), to two other constant vectors, namely $1-x$ and $\iota^{\prime}-x^{\prime}$, or to the axes $\mathbf{A B}, \mathbf{A B}$, of the two cylinders of revolution which can be circumscribed about the same ellipsoid. And the point of intersection of this other pair of opposite sides of the same inscribed quadrilateral is, by (123.), the extremity of the vector $p$, or the point $\mathrm{E}$ on the surface of the original ellipsoid; while the point $\mathrm{H}$, which has been already seen to be the intersection of the former pair of opposite sides of the quadrilateral, since it has, by (116.), its vector $\xi_{1}=-b^{2}$, is the reciprocal point, on the surface of that other and reciprocal ellipsoid, which was considered in article 61 ; namely the point which is, on that reciprocal ellipsoid, diametrically opposite to the point which was named $\mathbf{r}$ in that article, and had its vector $=b^{2}$.

67. Conversely it is easy to see, that the foregoing analysis by quaternions conducts to the following mode of constructing $\dagger$, or generating, geometrically, and by a graphic rather than by

* A Quaternion, geometrically considered, is the product, or the quotient, of any two directed lines in space.

+ This construction, of two reciprocal ellipsoids from one sphere, was comnunicated to the Royal Irish Acadeny in June 1848 ; together with an extension of it to a mode of generating two reciprocal cones of the second degree from one rectangular cone of revolution; and also to a construction of two reciprocal liyperboloids, whether of one sheet, or of two sheets, from one equilateral liyperboloid of revolution, of one or of two sheets. 
a metric process, a system of troo reciprocal ellipsoids, derived from one fixed sphere; and of determining, also graphically, for each point on either ellipsoid, the reciprocal point on the other.

Inscribe in the fixed sphere a plane quadrilateral $\left(L_{1} M_{l} L_{l}^{\prime} \mathbf{M}_{i}^{\prime}\right)$, of which the four sides $\left(\mathbf{L}_{i} \mathbf{M}_{l}, \mathbf{M}_{l} \mathbf{L}_{l}^{\prime}, \mathbf{L}_{l}^{\prime} \mathbf{M}_{l}^{\prime}, \mathbf{M}_{l}^{\prime} \mathbf{L}_{l}\right)$ shall be respectively parallel to four fixed right lines $\left(\mathbf{A B}, \mathrm{AC}^{\prime}, \mathrm{AB}^{\prime}, \mathbf{A C}\right)$, diverging from the centre (A) of the sphere; and prolong (if necessary) the first and third sides of this inscribed quadrilateral, till they meet in a point $\mathrm{E}$; and the second and fourth sides of the same quadrilateral, till they intersect in another point $\mathrm{H}$. 'Then these two points, of intersection $\mathrm{E}$ and $\mathbf{H}$, thus found from two pairs of opposite sides of this inscribed quadrilateral, will be two reciprocal points on two reciprocal ellipsoids; which ellipsoids will have a common mean axis, namely that diameter of the fixed sphere which is perpendicular to the plane of the four fixed lines: and those lines, $\mathbf{A B}$, $\Delta \mathrm{C}^{\prime}, \mathrm{AB}^{\prime}, \Lambda \mathrm{C}$, will be related to the two ellipsoids which are thus the loci of the two points $\mathrm{E}$ and $\mathrm{H}$, according to the laws enunciated in article $6 \mathrm{l}$, in connexion with a different construction of a system of two reciprocal ellipsoids (derived there from one common moring sphere); which former construction also was obtained by the aid of the calculus of quaternions. Thus the lines $\mathrm{Ac}, \mathrm{Ac}^{\prime}$ will be the two cyclic normals of the ellipsoid which is the locus of $\mathrm{E}$, but will be the axes of circumscribed cylinders of revolution, for that reciprocal ellipsoid which is the locus of $\mathrm{H}$; and conversely, the lines $A B, A B^{\prime}$ will be the axes of the two cylinders of revolution circumscribed about the ellipsoid ( $\mathbf{s}$ ), but will be the cyclic normals, or the perpendiculars to the cyclic planes, for the reciprocal ellipsoid $(\mathbf{H})$.

[To be continued.]

\section{Notices respecting Nerw Books.}

Letters addressed to H.R.H. the Grand Duke of Saxe Coburg and Gotha, on the Theory of Probabilities, as applied to the Moral and Political Sciences. By M. A. Quetelet, Astronomer Royal of Belgium, Corresponding Member of the Institute of France, \&c. \&c. Translated from the French by Olinthus Gregory Downes, of the Economic Life Assurance Society.

( F this work, which was begun by $M$. Quetelet in 1837, and published at Brussels, we believe, carly in 1845, the author thus describes the object in his preface.

"Certain circumstances, which have left me many pleasant reminiscences, made it necessary for me nearly ten years since to devote 